\author{
Filipe Caseiro-Alves \\ Jorge Brito \\ Antonio Eiras Araujo \\ Pedro Belo-Soares \\ Henrique Rodrigues \\ Augusta Cipriano \\ Daniel Sousa \\ Didier Mathieu
}

\section{Liver haemangioma: common and uncommon findings and how to improve the differential diagnosis}

Received: 31 January 2006

Revised: 15 September 2006

Accepted: 12 October 2006

Published online: 27 January 2007

(C) Springer-Verlag 2007
F. Caseiro-Alves $(\bowtie) \cdot J$. Brito ·

P. Belo-Soares · H. Rodrigues

Clínica Universitária de Imagiologia,

Hospitais da Universidade de Coimbra,

Praceta Mota Pinto,

3000-075 Coimbra, Portugal

e-mail: fca@netvisao.pt

Tel.: +351-239-400431

Fax: +351-239-482840

A. E. Araujo - D. Sousa

Department of Diagnostic Radiology,

University of Rio de Janeiro

and Labs-D'Or Hospital,

Rio de Janeiro, Brazil
A. Cipriano

Serviço de Anatomia Patológica,

Hospitais da Universidade de Coimbra,

Praceta Mota Pinto,

3000-075 Coimbra, Portugal

D. Mathieu

Centre de Radiologie,

1, Boulevard de la Republique,

13100 Aix en Provence, France

\begin{abstract}
Haemangiomas are common focal liver lesions, generally detected in the work-up of asymptomatic patients. From the pathological point of view, they can be classified as small (capillary) or large, with cavernous vascular spaces that may show thrombosis, calcifications and hyalinisation. The polymorphic imaging appearance of haemangiomas depends on their histological features and flow pattern. The widespread use of cross-sectional imaging has allowed an increased detection rate and a better characterisation of this benign
\end{abstract}

tumour. Recent developments of ultrasound (US), computed tomography (CT) and magnetic resonance imaging (MRI) providing high spatial and temporal resolution, together with the use of new contrast agents and/or pulse sequences has broadened the spectrum of imaging findings, contributing to diagnostic refinement in difficult cases. The scope of the present article is to provide an overview of the range of appearances of haemangiomas, explored with recent crosssectional imaging modalities, emphasising its atypical findings as explored by temporally resolved contrast-enhanced imaging.

Keywords Ultrasound · Computed tomography $\cdot$ X-ray computed . Magnetic resonance imaging - Liver . Neoplasms · Haemangioma ·

Contrast agents

\section{Introduction}

Currently, the widespread use of modern cross-sectional imaging methods has led to an increased fortuitous detection of focal liver lesions in all patients, including the asymptomatic and oncologic groups. Since imaging techniques can now provide high spatial resolution, small liver nodules are easily depicted, posing the problem of the characterisation of the so-called incidental lesions. Recent papers based on helical computed tomography (CT) or on necropsy studies showed that liver tumours are more frequent than it was previously accepted [1]. Among those, the majority correspond to cysts, haemangiomas, focal nodular hyperplasia, adenomas and pseudolesions, such as focal fatty infiltration and focal fatty sparing [2]. It has been demonstrated that the majority of incidental focal liver lesions discovered during any abdominal imaging work-up is, in fact, benign and devoided of particular clinical significance, even in oncologic patients [3, 4].

The characterisation of benignancy, when dealing with a focal liver lesion, assumes special importance, since it leads to cost reduction concerning further imaging work-up 
and spares the patient from anxiety. Several factors are implicated on the confidence level assigned to characterise a focal liver lesion, such as their typical or atypical imaging findings, the previous existence of an oncologic disease, de novo appearance or modification of the imaging features as seen on follow-up studies. Diminutive size is one of the major constraints to achieving a proper characterisation and even the state-of-the-art imaging techniques may fail to provide the diagnosis in such cases, ultimately leading to other diagnostic tests, possibly even invasive methods [5].

The scope of this paper is to review the atypical features of liver haemangiomas and to show how to improve the diagnostic confidence in their characterisation by using state-of-the-art cross-sectional imaging techniques.

\section{General considerations}

Haemangiomas have an estimated prevalence of $0.4 \%$ to $20 \%$, according to several autopsy studies. Being devoid of malignant potential, they are, in general, fortuitously discovered during any abdominal imaging work-up, mostly being discovered between the fourth and fifth decades of life [5-7]. They are multiple in more than $50 \%$ of cases and show a clear female predilection (ratio of 2-5:1).

Pathological examination (Fig. 1a) reveals a focal tender mass formed by multiple vascular channels limited by a single layer of endothelial cells within a thin fibrous stroma. In general, the blood circulation within these tumour vessels is slow. Morphologically, it is a welldefined lesion, possessing round or lobulated margins. Although it may occur anywhere in the liver parenchyma, it is more commonly seen at peripheral and sub-capsular locations, mostly in the posterior segments of the right lobe $[5,6,8]$. Their size usually remains stable and can vary from a few millimetres to more than $20 \mathrm{~cm}$, a feature that has been used for its classification into small $(<15 \mathrm{~mm})$, medium $(15-50 \mathrm{~mm})$ and large haemangiomas $(>50 \mathrm{~mm})$ [6]. Large haemangiomas may give rise to complications in $4.5 \%$ to $19.7 \%$ of cases, consisting of bleeding, compressive effect on adjacent structures such as the bowel and torsion if pedunculated $[6,7,9,10]$.
Fig. 1 a-d Usual findings in haemangiomas. a Histological feature depicting the large vascular spaces of a well-defined non-capsulated tumour. b On ultrasound (US), the tumour is homogeneously hyperechoic with lobulated contours. c On computed tomography (CT), centripetal, progressive enhancement is seen on the arterial phase of the dynamic liver study. d Magnetic resonance imaging (MRI) shows a homogeneous lesion with intensity similar to cerebrospinal fluid (CSF) on T2-w fatsat
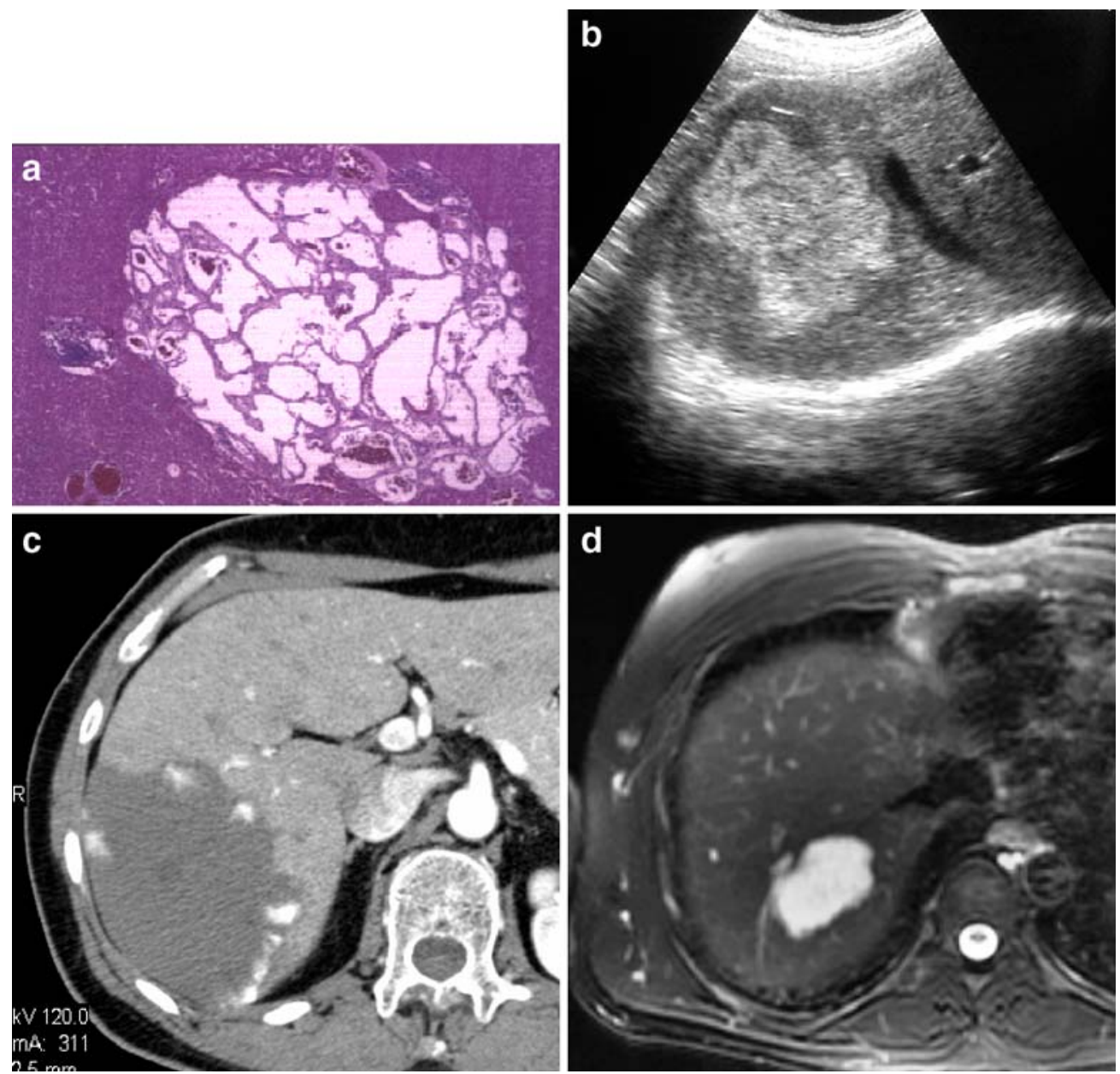
Fig. 2 a, b A case illustrating the possibility of differentiating non-invasively haemangiomas from cysts using plain MRI Compared to the T2-w fatsat fast spin echo (FSE) sequence (a), the haemangioma shows a considerable signal intensity loss on the steady-state free precession (SSFP) images (TrueFISP) (b). Contrarily, biliary cysts retain high signal intensity on both sequences
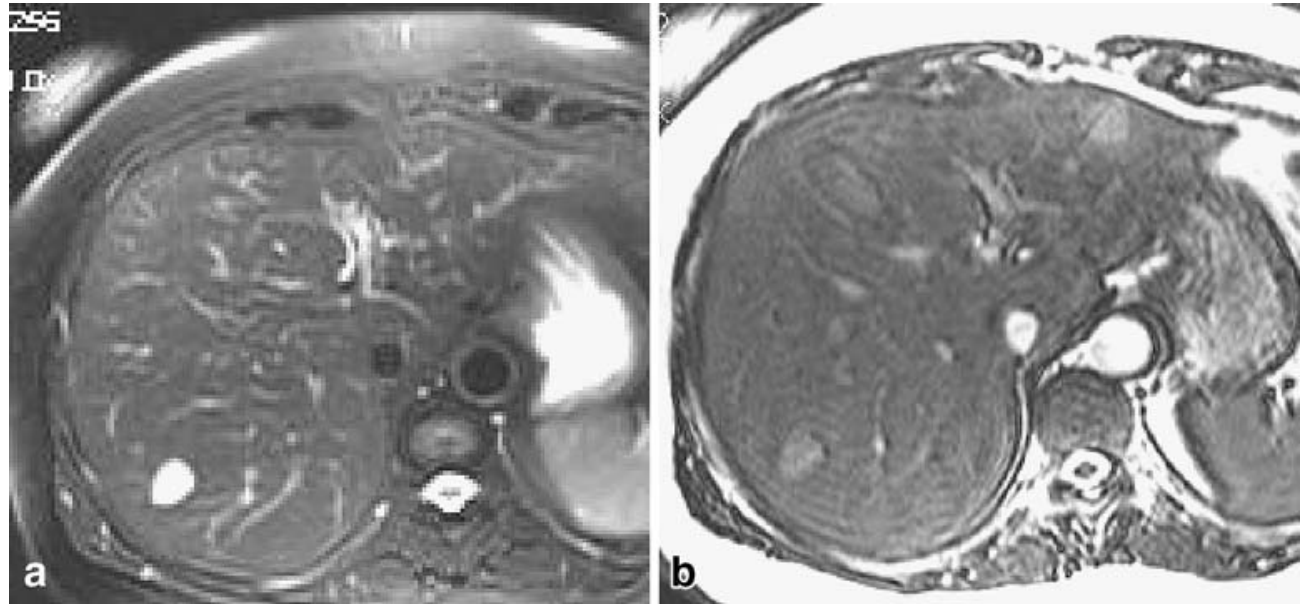

\section{Common imaging findings}

The most common sonographic pattern of haemangiomas (Fig. 1b) consists of the demonstration of a homogeneous hyperechoic liver nodule, with discrete posterior acoustic enhancement, devoided of Doppler signal both on colourcoded and spectral examination [11].

CT features (Fig. 1c) usually shows a hypodense welldefined lesion, with an internal density similar to the vessels. Dynamic studies performed after the administration of non-specific extracellular iodine compounds exhibits early nodular peripheral enhancement (puddling), since its feeding vessels originate from the hepatic artery. Subsequent phases of liver enhancement reveal a progressive slow centripetal fill-in, with iso- or even slight hyperdensity on delayed phase as compared to the normal liver parenchyma. This classical presentation is diagnostic with a high level of confidence $[5,6]$. Late intra-tumoural accumulation of contrast can be explained by the slow flowing blood within its vascular channels determining iso-/hyperdensity and absence of noticeable washout on the latter phases of the dynamic study $[5,7,12,13]$. However, delayed homogeneity due to complete contrast filling of the tumour should not be regularly expected, especially for large tumours, and, thus, should not be used as mandatory diagnostic criteria. Small haemangiomas may appear to enhance to a lesser extent but this may result from partial volume averaging $[5,12]$.

Currently, magnetic resonance imaging (MRI) is the imaging method of choice to diagnose haemangiomas (Fig. 1d) [5, 6]. On T1-weighted (-w) images, haemangiomas display low signal intensity and on $\mathrm{T} 2-\mathrm{w}$, they are strikingly homogeneous, clearly demarcated from the adjacent parenchyma, with very high signal intensity,
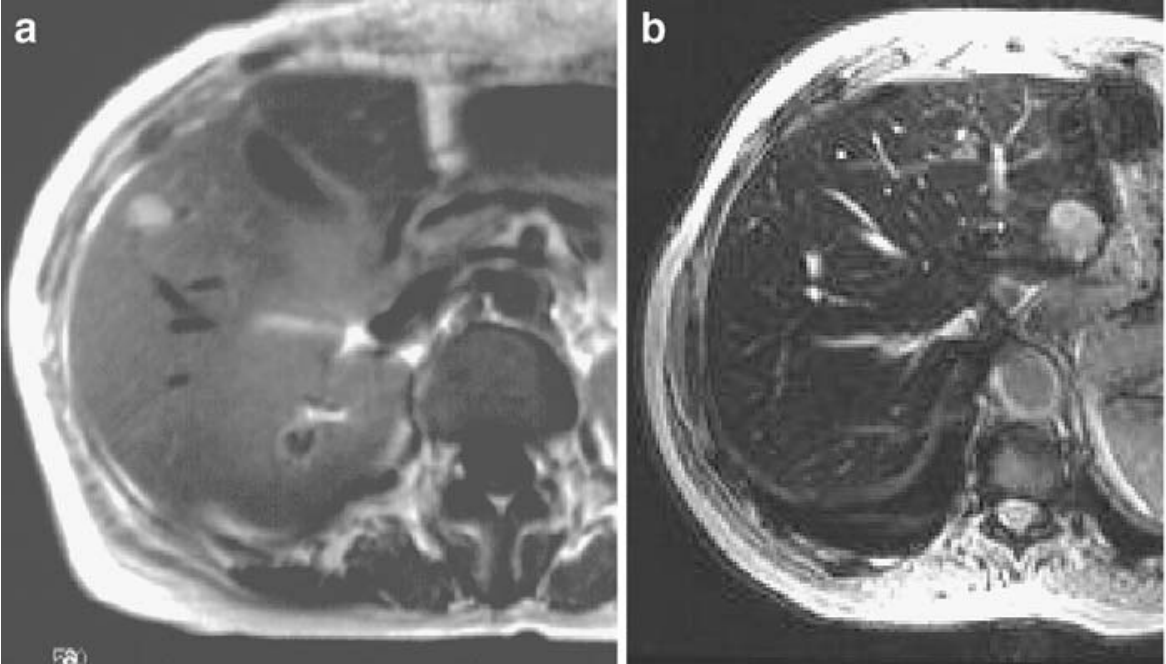

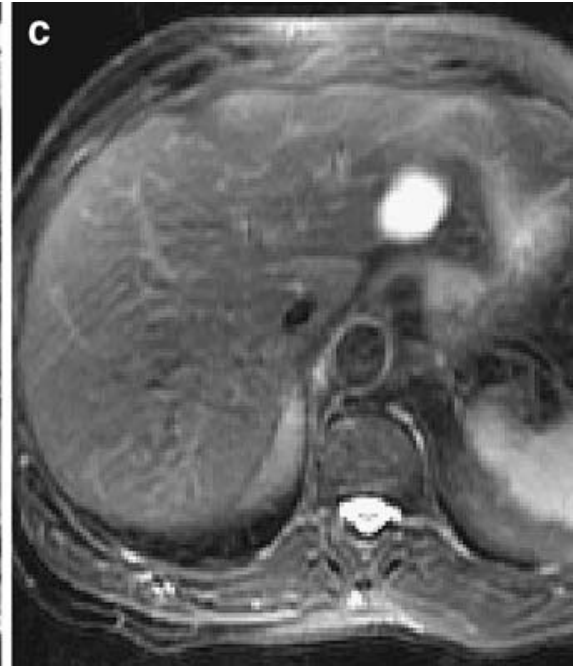

Fig. 3 a-c Haemangiomas and iron-oxide enhanced MRI. a Ironoxide enhanced T1-W images obtained in the late retention phase. The small haemangioma of the right liver lobe becomes hyperintense, which can be explained by the T1-effect exerted by the intra-tumoural circulating iron oxide particles. Another case showing signal intensity loss on the retention phase of the postcontrast GRE T2-w image (b) compared to the plain T2-w fatsat image (c) 
Fig. 4 a Isoechoic haemangioma with a hyperechoic border; this is a common atypical appearance that has been associated with internal necrosis, scarring or myxomatous changes. b Pathological specimen of a cavernous haemangioma with a central area of myxomatous degeneration (arrow). c Haemangioma on a T2-w image displaying lobulated contours and a hyperintense central scar. d MRI features of a giant cavernous haemangioma; the tumour is heterogeneous, displaying internal septations, lobulated contours and a CSFlike signal intensity on this FSE T2-w image

Fig. 5 a T2-w image of a peripheral haemangioma of the right liver lobe displaying capsular retraction. Signal intensity on the T2-w images and the contrast enhancement pattern (not shown) were sufficient to warrant the diagnosis. b Coronal volume-rendered (VR) image of a contrast-enhanced multi-detector computed tomography (MDCT) study showing a large pedunculated haemangioma originating in the left liver lobe
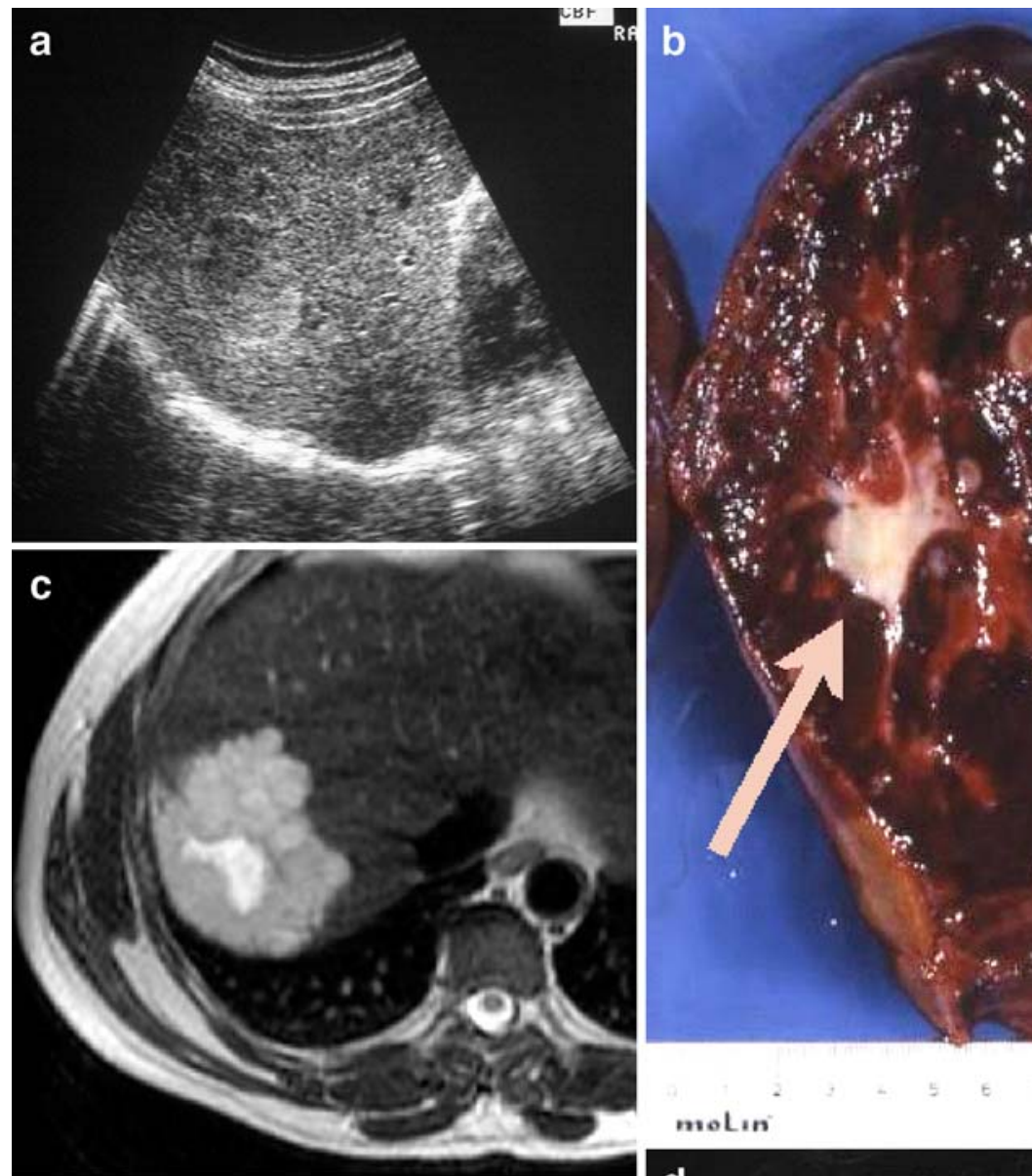

$\infty$

$x^{2}$

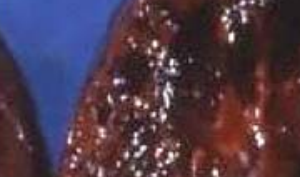

7. $\%$

is:
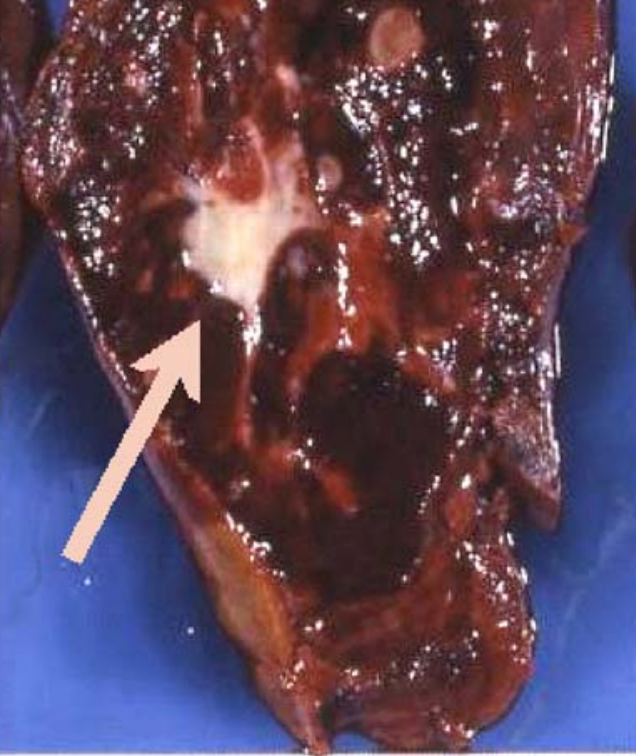

molin
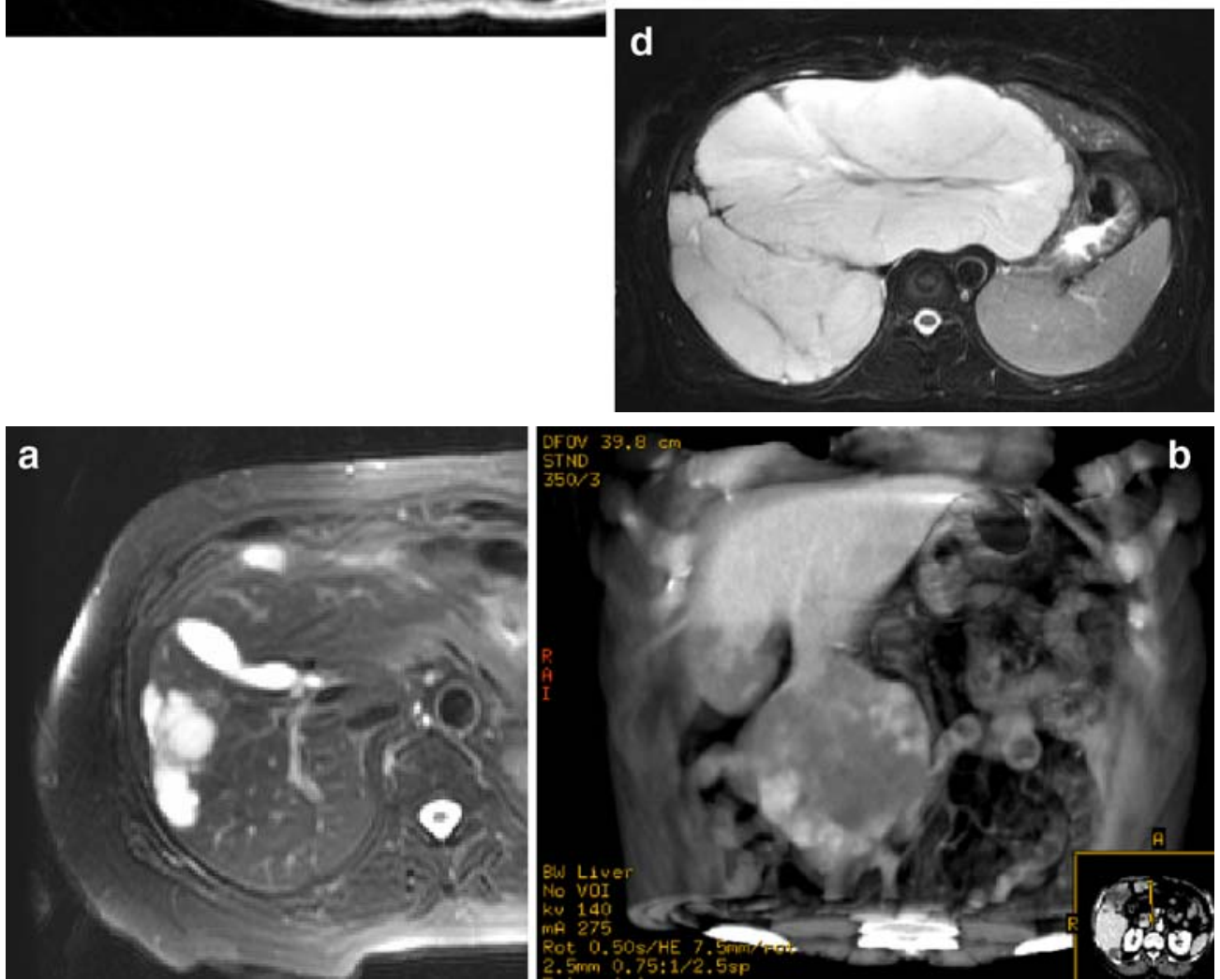
Fig. 6 a, b Asymptomatic haemangioma causing bile duct dilatation. a Contrast-enhanced CT study depicting a segment IV haemangioma. b Slice obtained at an upper level revealing upstream bile duct dilatation caused by extrinsic compression. This finding should not preclude the diagnosis. In this case, imaging findings remain stable 4 years after the initial diagnosis

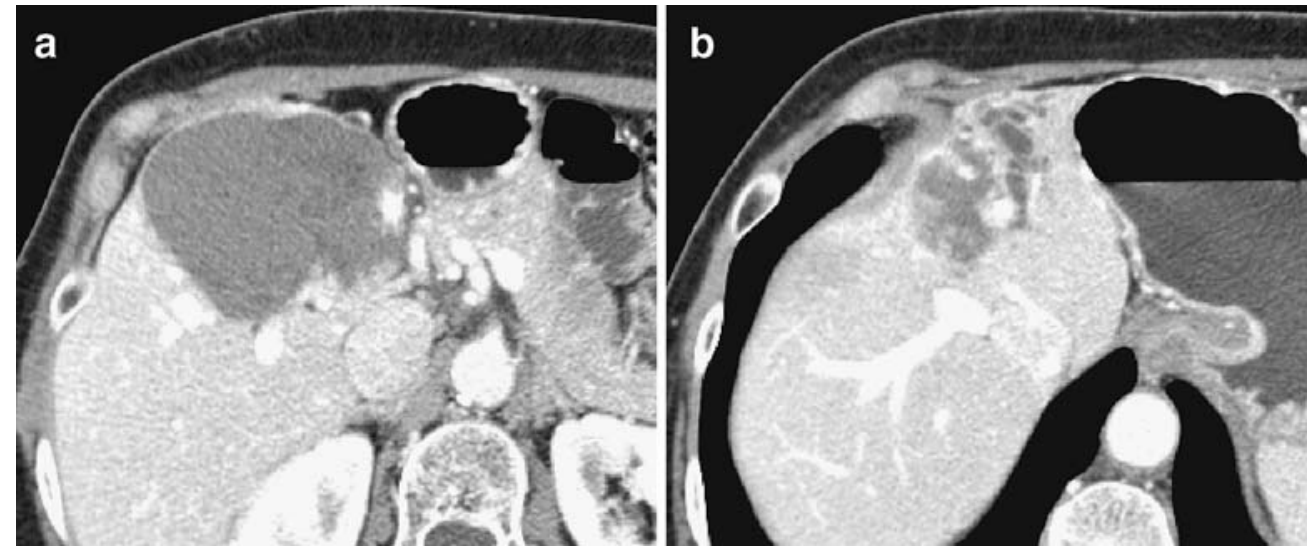

similar to cerebrospinal fluid (CSF), due to the long T2 relaxation time of its blood-filled vascular channels, a feature that has been coined as the bulb-light sign $[5-7,10]$. In the past, calculation of the T2 relaxation time was deemed useful for its differential diagnosis with other focal liver lesions, since a relaxation value of at least $112 \mathrm{~ms}$ could differentiate it from metastases with a 92-97\% accuracy $[5,6,12,14-16]$. Other MRI approaches may include a double-echo heavily $\mathrm{T} 2-\mathrm{w}$ sequence $[17,18]$, a fluid-attenuated inversion recovery (FLAIR) or the use of the balanced steady-state free precession (SSFP) sequence $[19,20]$, which can discriminate between haemangiomas and simple liver cysts without the need for contrast injection (Fig. 2). More recently, a diffusion-weighted echo-planar technique has demonstrated usefulness in distinguishing benign from malignant liver tumours, based on their different magnetic properties at a microscopic level [21].

The high spatial and temporal resolution provided by state-of-the-art MRI equipment is currently used to acquire fast gradient-echo imaging (GRE) within the time frame of a single breath-hold, allowing contrast-enhanced dynamic liver imaging using gadolinium chelates. Characterisation of focal liver lesions are, thus, being progressively obtained by the combined information conveyed by static and dynamic imaging, with haemangiomas displaying similar dynamic MRI features as seen on CT. Liver MRI can also be performed with dedicated cell-specific contrast agents, such as iron-oxide particles (SPIO), which are captured by the Kupffer cells of the reticulo-endothelial system. Using the most recent iron-oxide contrast agents, compatible with a bolus-injection of small volumes, haemangiomas can display T1-w enhancement, due to the initial extra-celular distribution of the SPIO agent, similar to that observed with gadolinium chelates. In the late retention phase, haemangiomas can also show peculiar imaging features consisting of discrete hyperintensity on T1-w images (Fig. 3a). This is due to the slow intra-tumoural blood flow carrying iron-oxide particles which, at very low concentrations, produce shortening of the tumour T1 relaxation time. On T2-w sequences, which are more prone to be disturbed by magnetic susceptibility effects caused by the iron-oxide particles, the haemangioma may show negative enhancement and, consequently, signal loss, which may pose an additional difficulty for its characterisation $[6,22,23]$ (Fig. 3b,c). Since haemangiomas do not contain hepatocytes, enhancement should not be expected with other specific paramagnetic contrast agents and their imaging features are basically unremarkable when compared to other hepatocyte-devoided liver tumours [6].

Recently, pulse-inversion ultrasound (US) after administration of the second-generation contrast agents replicates the characteristic puddling and progressive centripetal fillin of haemangiomas, contributing to a diagnostic refinement for undetermined cases seen on the baseline B-mode US [24-26].

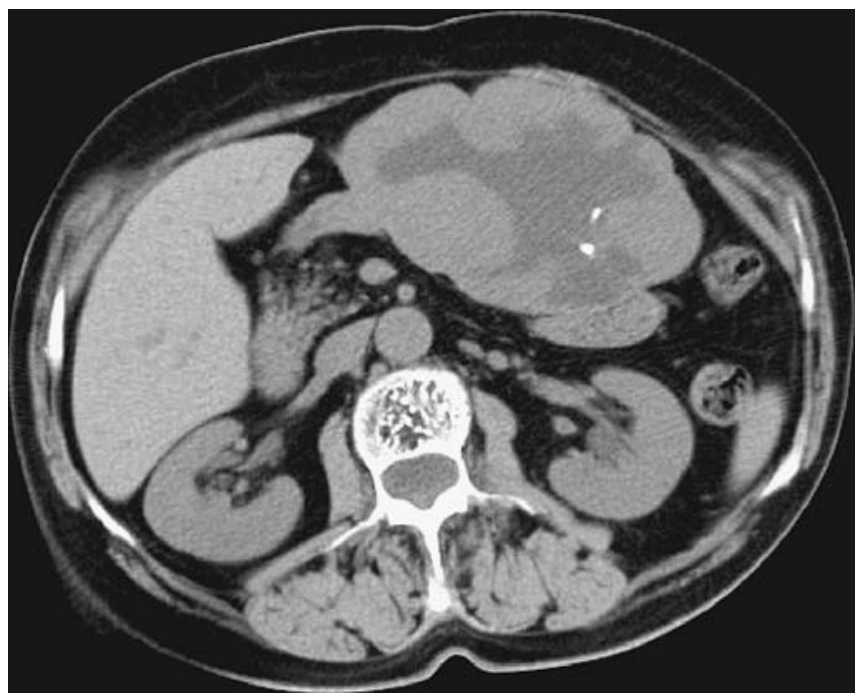

Fig. 7 Plain CT of a cavernous haemangioma showing punctate calcifications. In general, calcifications are due to intra-lesional phlebolites but may be the end result of fibrosis and dystrophic changes 
Fig. 8 a, b Haemorrhagic haemangioma seen on MRI. a T1-w FSE image disclosing an internal hyperintense area. b On the corresponding T2-w FSE image, the haemorrhagic area is hypointense, which is due to the susceptibility effects caused by the methaemoglobin content. Other areas of scarring and fibrosis are also apparent, exhibiting low signal intensity on both sequences. c T2-w fatsat image of a haemangioma with a fluid-fluid level. Very rarely, haemangiomas can display an internal fluid-fluid level traducing different blood flow velocities within the haemangioma, with layering of red blood cells on the dependent portion
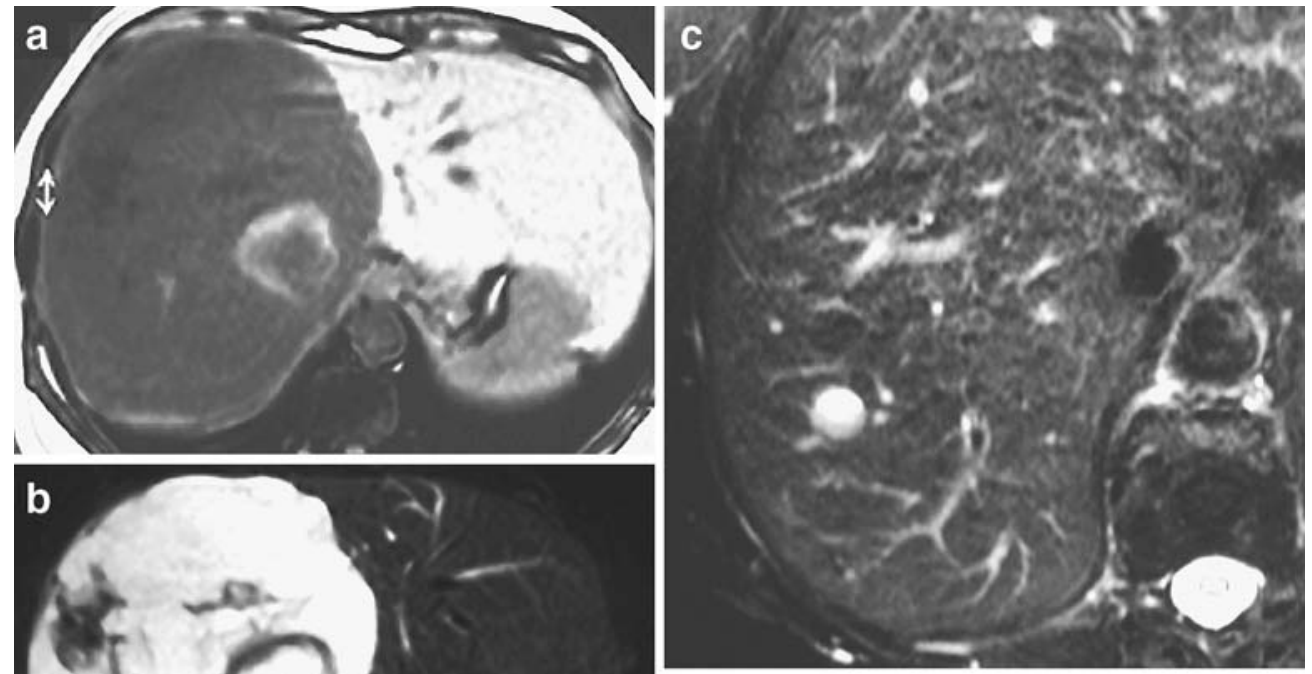

\section{Uncommon presentations of haemangiomas}

The diagnostic management of haemangiomas depend both on the clinical context and the imaging features of the tumour. In patients with a known malignancy, and with previous normal examinations, or in cases of atypical imaging presentation, the diagnosis needs to be confirmed by other means beyond US, ultimately leading to the use of invasive techniques [11]. Less typical imaging findings of haemangiomas can result from three main causes: altered morphology or structure, unusual flow patterns or associated liver abnormalities. Although these aspects do not preclude in all instances the correct diagnosis, they may be misleading and, therefore, should be known by radiologists in order to avoid other potentially more invasive diagnostic tests. Whenever the diagnosis is doubtful, especially in oncologic patients, even after a multimodality imaging approach, a percutaneous biopsy could be sought, with considerable evidence concerning its safety [27].

\section{Uncommon morphology/structure}

On grey-scale US, atypical haemangiomas can show an internal iso-/hypoechoic texture with a peripheral hyperechoic border (Fig. 4a), which has been associated with internal thrombosis or scarring [7]. Large haemangiomas are frequently heterogeneous and may display a central scar (Fig. 4b,c), which does not enhance on the late interstitial phases of the dynamic studies. On pathology, the scar is formed by myxomatous degeneration, thrombosis, fibrosis or necrosis [6]. The T2-w images are frequently unable to determine its exact composition, as seen on pathological studies, because a central fluid-like hyper-

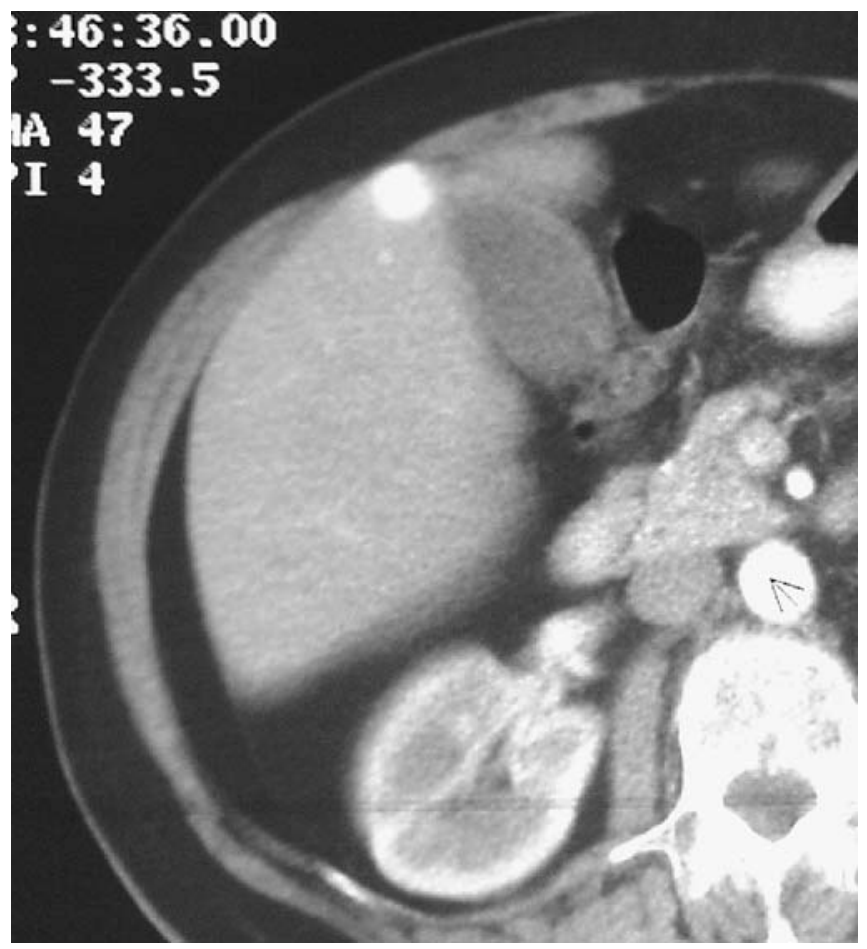

Fig. 9 Flash-filling haemangioma on dynamic contrast-enhanced CT showing intense homogeneous blush on the arterial phase. Isodensity was observed on the delayed image (not shown) 

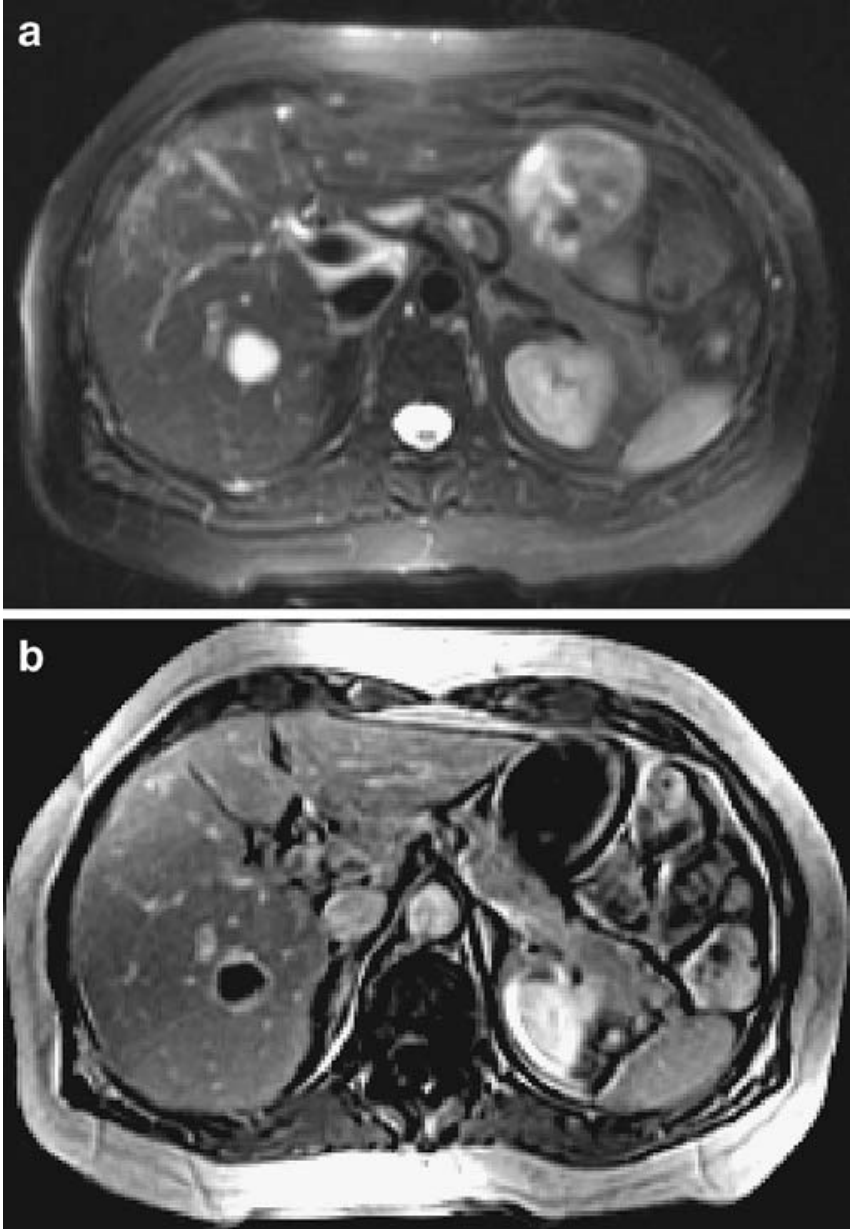

Fig. 10 a, b Hypodynamic haemangioma with a late-filling pattern on MRI. a Plain T2-w image showing high signal intensity and homogeneous pattern. b Dynamic imaging showing peripheral enhancement on the portal phase of the dynamic study. Thirty minutes after contrast injection, the fill-in pattern and persistent enhancement were seen (not shown), thus, enabling the correct diagnosis intensity can be displayed, even in cases of myxomatous degeneration. Other atypical morphological features consist of the presence of internal septations (Fig. 4d), exhibiting low signal intensity on T1- and T2-w images related to the presence of a fibrotic component.

Liver capsule retraction is not a frequent finding, but it should not preclude the diagnosis, since it may be seen as secondary to peripheral fibrotic changes (Fig. 5a) $[7,28$, 29]. In this case, other diagnoses should be ruled out, such as intra-hepatic cholangiocarcinoma, epithelioid haemangioendothelioma or, more rarely, metastases with a fibrotic component. Pedunculated haemangiomas are rare and, in general, its signal intensity and enhancement pattern is consistent with the diagnosis (Fig. 5b), except if they undergo torsion and infarction, when they can become symptomatic. Recognition of its hepatic origin is better accomplished using multiplanar images, allowing identification of the stalk [7, 30]. Sometimes, bile duct dilatation may occur because of large haemangiomas centrally located within the liver, especially around segment IV and near the liver hilum (Fig. 6). Therefore, this feature should not preclude the diagnosis [7, 30, 31].

Calcifications in haemangiomas can be found with a central or peripheral location, with multiple foci usually representing phlebolites. They are better depicted on CT as tiny dense nodules or as low signal intensity foci within the hyperintense tumour on T2-w images [7] (Fig. 7). MRI is useful for identifying intra-tumoural haemorrhage, displaying areas with high signal on T1-w images, best depicted on fat-supression sequences (Fig. 8a,b). Blood signal intensity on $\mathrm{T} 2-\mathrm{w}$ images is variable, depending on the products of haemoglobin degradation. The spared nonhaemorrhagic areas of the lesion usually maintain the same signal intensity and enhancement pattern of typical haemangiomas [7]. Intra-peritoneal bleeding is exceedingly rare but may be associated even with minor
Fig. 11 a, b Hyperdynamic haemangiomas seen on dynamic $\mathrm{CT}$ associated with an arterioportal fistula. a Plain image disclosing four hypodense focal lesions at the right liver lobe. b The arterial phase of the dynamic CT study reveals two haemangiomas with the usual flow pattern and two other flash-filling, causing adjacent perfusion abnormalities. Early opacification of a segmental branch of the portal vein (arrow in b) can be recognised due to an associated arterio-portal fistula. An incidental liver cyst is seen at segment IV
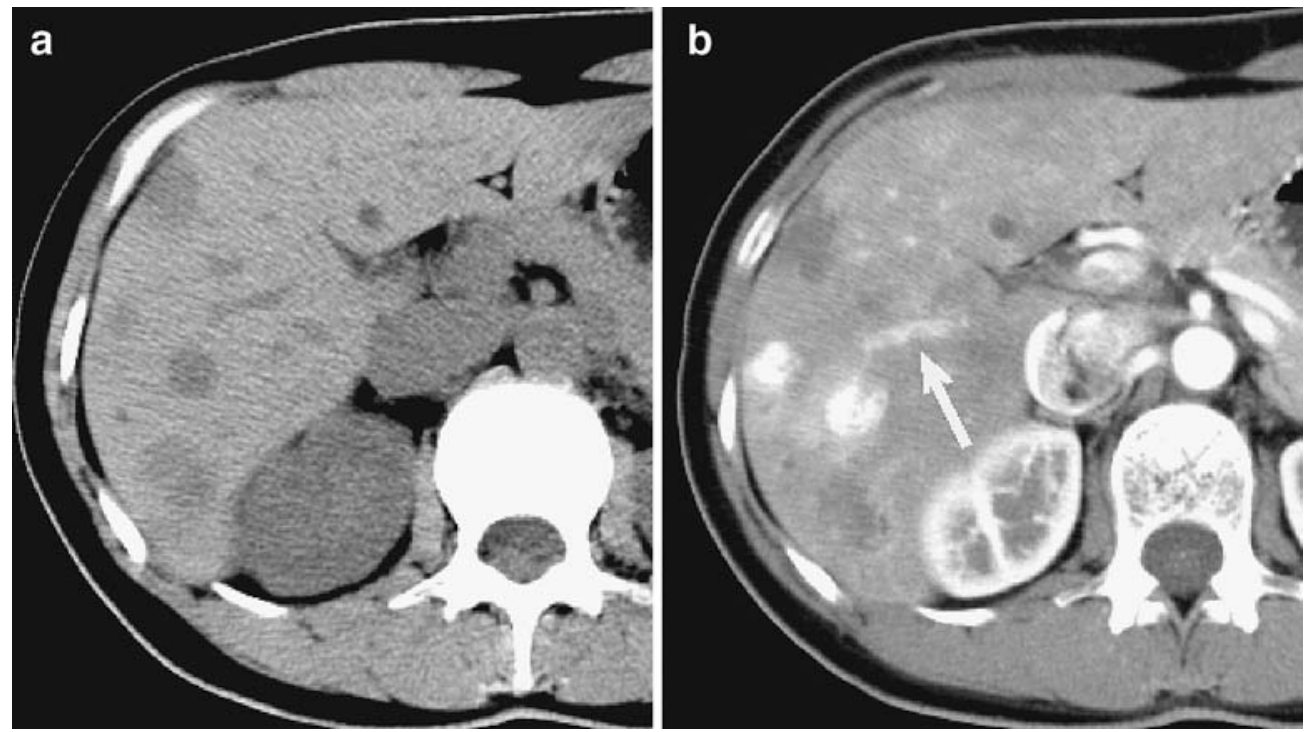
Fig. 12 a, b Other atypical enhancement patterns of haemangiomas seen on CT. a Arterial phase image of a dynamic CT study showing a triangular hyperdense area representing arterio-venous shunting. This area corresponds to a transient hepatic attenuation differences (THAD) that was no longer depicted in the portal venous phase (not shown). b Haemangioma showing an area of central enhancement
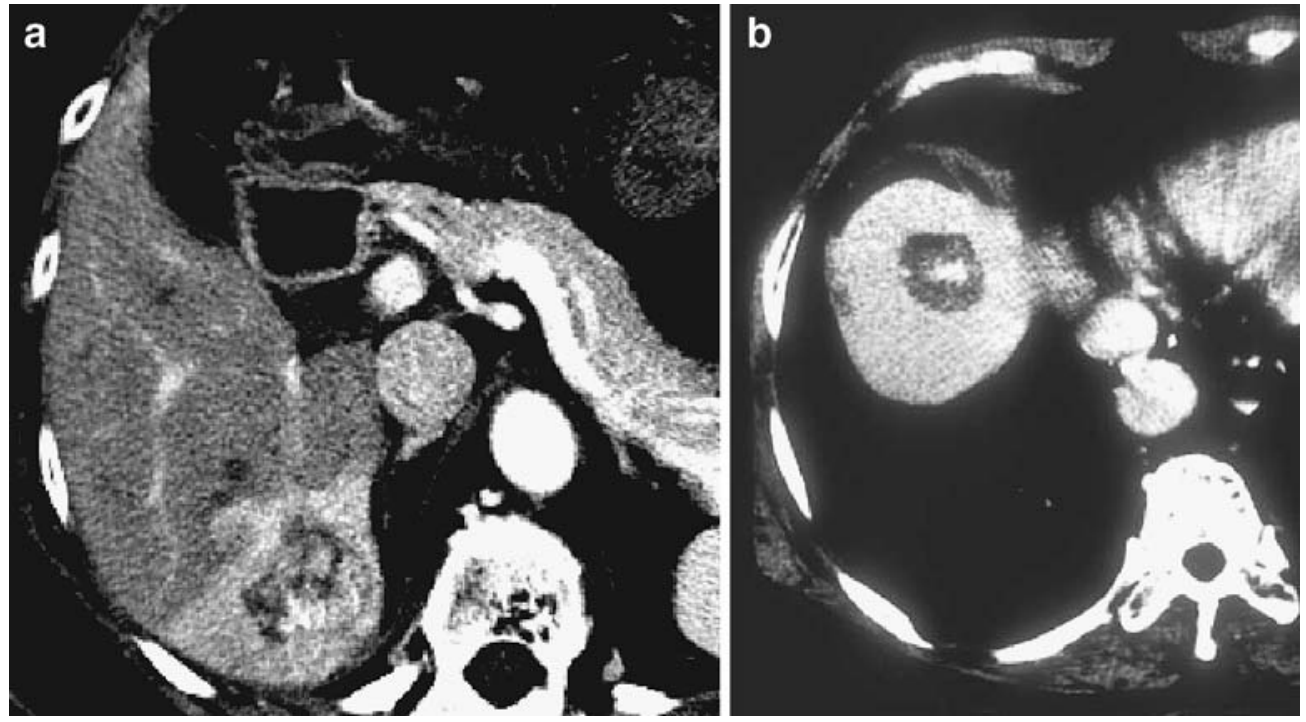
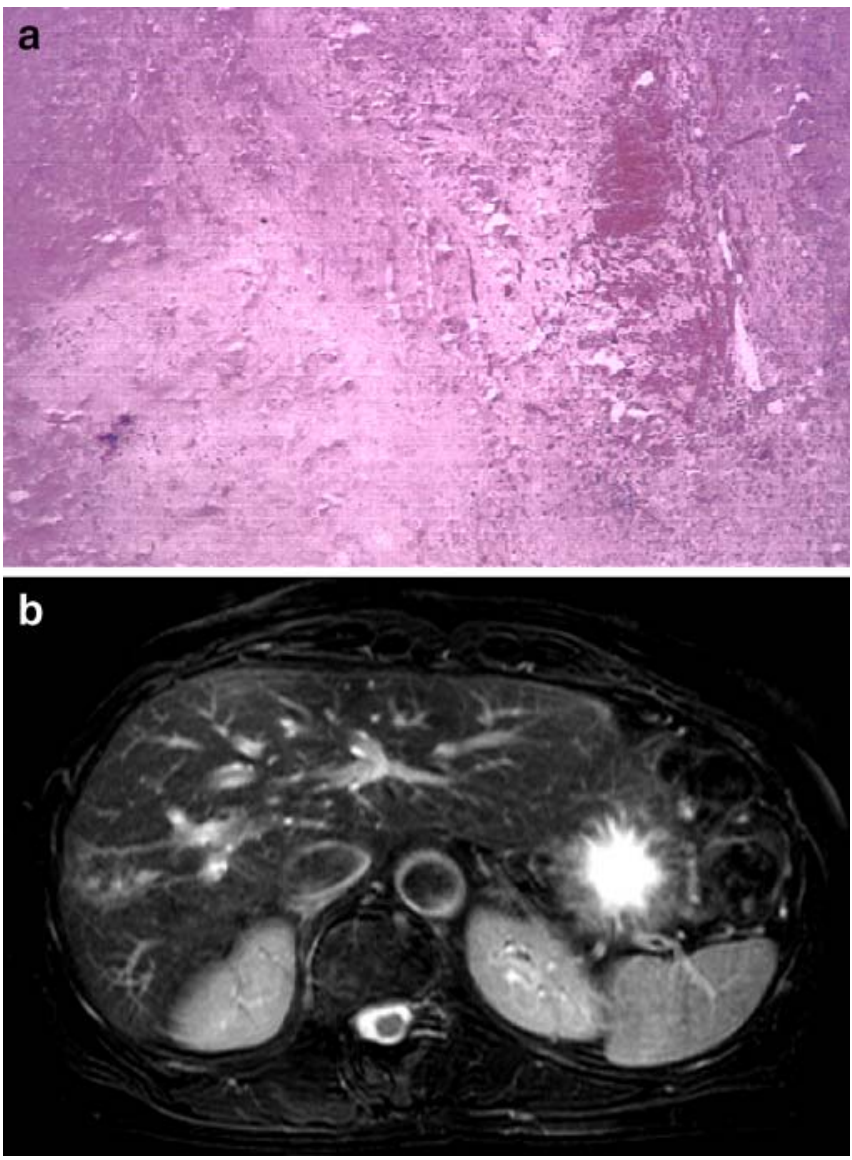

Fig. 13 a, b Sclerosed haemangioma. a Low-power magnification haematoxylin and eosin (H\&E) staining showing complete obliteration of the vascular channels and replacement by fibrous tissue. $\mathbf{b}$ Corresponding heavily $\mathrm{T} 2 \mathrm{-w}$ image disclosing loss of the typical light bulb intensity of the haemangioma (compare with the CSF signal) abdominal trauma in cases of large superficial tumours. Cystic and multilocular haemangiomas containing a large central cavity are extremely rare. On MRI, this atypical feature is represented by a single or multiple intra-tumoural cavities, possessing long $\mathrm{T} 1$ and $\mathrm{T} 2$ relaxation times related to thrombosis and old haemorrhage. Nevertheless, peripheral enhancement with puddling can still occur [7]. In rare instances, a fluid-fluid level may be depicted inside the haemangioma, which is thought to represent stagnant or slow flowing blood with sedimentation of red blood cells on the dependent portion [32]. The superior fluid layer consists of serum and the inferior layer contains unclotted sedimentary red blood cells $[7,32]$. On CT, the superior layer shows fluid attenuation, while the inferior layer has higher attenuation due to the density of the packed cells. On MRI, T2-w sequences show high signal intensity on the upper layer and a less intense signal on the more cellular inferior layer (Fig. 8c). The fluid-fluid level is not specific of haemangiomas and has been reported in other lesions, such as complicated cysts, ciliated foregut cysts, chronic haematomas, biliary cystadenomas, tumours with liquefactive necrosis or haemorrhage, and necrotic metastases [33]. In general, histopathologic correlation is necessary to confidently establish the diagnosis [7, 32-34].

Interval growth has been reported in haemangiomas, although it is extremely rare. In this situation, the initial diagnosis should be questioned and biopsy studies performed on selected cases [9].

\section{Uncommon enhancement patterns}

The current use of state-of-the-art CT and MRI equipment with high temporal resolution increasingly reveals less known enhancing patterns of haemangiomas. The so-called flash-filling pattern is mostly seen in small tumours under 
Fig. 14 Haemangioma and associated liver abnormalities. a, b Haemangioma in fatty liver. a Plain CT image disclosing a low liver density consistent with diffuse steatosis. The haemangioma is practically invisible. b After contrast enhancement, the haemangioma is disclosed with the typical peripheral globular enhancement. c, d Association of focal nodular hyperplasia $(\mathrm{FNH})$ and haemangioma. c Arterial phase of a dynamic MRI depicting the vigorous enhancement of an FNH at the left liver lobe and the peripheral puddling of contrast in the small haemangioma at segment VII d Portal venous phase of the same case on CT showing contrast retention within the haemangioma and a nearisodensity of the left lobe FNH
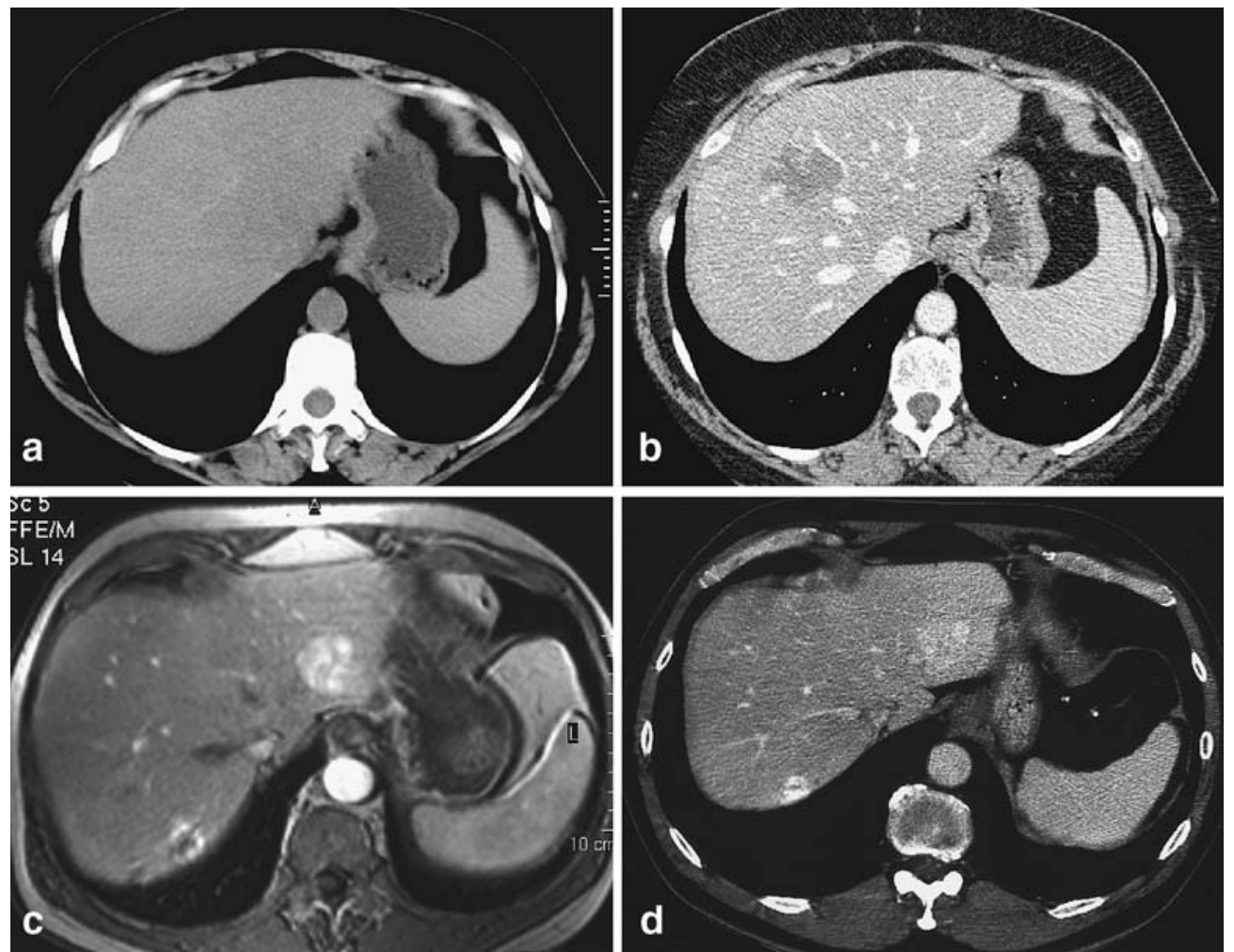

$2 \mathrm{~cm}$ and show fast, intense and uniform enhancement on the arterial phase of the dynamic study, typically, paralleling aortic enhancement (Fig. 9) [35, 36]. Since these tumours may also display colour spots on Doppler examination, differentiation from other hypervascular tumours may be problematic. A key point resides on the evaluation of the late interstitial phase of the dynamic study, where flash-filling haemangiomas should not reveal contrast washout. In contrast to haemangiomas with this type of hyperdynamic status, on the other side of the spectrum, hypodynamic tumours with very slow internal flow may be seen, resulting in a very delayed fill-in pattern. These cases request prolonged examination times with ultra-delayed acquisitions, in order to be able to observe the centripetal enhancement and contrast retention within the tumour (Fig. 10). These haemodynamic differences are probably related to the size of the vascular channels composing the haemangioma, with slow flow in areas of large channels and hyperdynamic flow in those containing small-sized channels.

Peri-lesional enhancement is seen in $19-25 \%$ of cases and manifests itself as transient hepatic attenuation differences (THAD), corresponding to staining areas seen on the arterial phase of liver enhancement, fading away on the subsequent phases of the dynamic study [36, 37]. Pathological explanation resides in arterio-venous shunting related to their hyperdynamic status. Early opacification of a drainage vein may occur (Fig. 11) [36, 38]. Although
THAD is mostly seen in cases of small flash-filling haemangiomas, larger tumours can also show the same perfusion abnormalities due to portal vein compression and compensatory arterial inflow, determining a peri-tumoural area of parenchymal enhancement [36] (Fig. 12a). It must be stressed that peri-lesional THADs have been described in association with a variety of focal liver lesions, such as metastases, abscesses and hepatocellular carcinoma [38]. Haemangiomas presenting an early central enhancement evolving centrifugally are rare (Fig. 12b). According to Kim et al. [13], these lesions have a central area with multiple vascular spaces and peripheral regions with a dominant fibrous component. More frequently, central enhancement is simulated by the axial nature of the CT slices.

Hyalinised or sclerosed haemangioma is rather unusual and is believed to represent the end stage of a haemangioma involution. The replacement of the vascular spaces by hyalinised fibrotic tissue leads to marked modifications of its imaging features, with loss of the typical globular enhancement on contrast-enhanced studies and of its high signal intensity on T2-w images on MRI (Fig. 13). Since it is virtually impossible to propose a definitive diagnosis in such a case, pathologic proof may be necessary, which discloses extensive fibrotic tissue and obliteration of the vascular channels. Differential diagnosis should include hypovascular tumours, especially metastases $[7,39]$. 
Associated liver abnormalities

Haemangioma in fatty liver may be difficult to diagnose on US and CT, due to the decreased liver-lesion contrast which, in severe steatosis, may even be reversed in both imaging modalities, with the haemangioma being hypoechoic and denser, respectively, when compared to the adjacent fatty liver (Fig. 14a,b). Although dynamic CT is able to show the characteristic enhancement pattern, MRI may be preferable in this setting, especially using in-phase and out-of-phase gradient-echo sequences. The high signal intensity on T2-w images and the enhancement features are preserved, thus, rendering the diagnosis relatively straightforward. Sometimes, in CT studies, a peripheral dense halo may be seen surrounding the haemangioma. This corresponds to spared non-fatty parenchyma, as a consequence of the preferential arterial inflow and/or decreased portal flow around the lesion due to compression phenomena [12].

Haemangioma in cirrhotic liver is rare, with an incidence estimated to be about $1.7 \%$ at pathologic examination and $0.6 \%$ at CT, which is clearly lower than the frequency in unselected autopsy series or at CT in non-cirrhotic patients [40,41]. Despite the stiffening of the liver parenchyma, haemangiomas generally retain their characteristic findings, both on $\mathrm{T} 2-\mathrm{w}$ images and the enhancement pattern. In rare cases, however, mostly in advanced cirrhosis, they can lose their typical imaging features due to the development of fibrosis, determining lower volume and lower signal intensity on T2-w images [12, 40, 42]. MRI seems to be superior to CT for characterising haemangiomas in cirrhosis, due to its intrinsic higher contrast resolution and the additional information conveyed by the $\mathrm{T} 2-\mathrm{w}$ sequences, which can also incorporate quantitative data as T2 relaxation value calculation and/or performance of sequences using very late echo times [40].

Haemangioma associated with focal nodular hyperplasia $(\mathrm{FNH})$ (Fig. 14c,d) has been reported in approximately $20 \%$ of cases of patients having a concomitant $\mathrm{FNH}$, a prevalence that is higher than that observed in the general population. A common physiopathological mechanism may explain this association, which is believed to result from a focal disturbance of the liver blood supply facilitating a hyperplastic response and subsequent development of these benign tumours [43, 44].

In conclusion, liver haemangiomas have specific imaging features, allowing its characterisation with cross-sectional imaging techniques. There is, however, a wide range of findings that fall out of the typical description, which are, essentially, due to modifications of the tumour structure or flow dynamics. Current stateof-the-art imaging techniques are more prone to demonstrating these findings, thus, increasing their diagnostic confidence level.

\section{References}

1. Völk M, Strotzer M, Lenhart M, Techert J, Seitz J, Feuerbach S (2001) Frequency of benign hepatic lesions incidentally detected with contrast-enhanced thin-section portal venous phase spiral CT. Acta Radiol 42(2):172-175

2. Bleuzen A, Tranquart F (2004) Incidental liver lesions: diagnostic value of cadence contrast pulse sequencing (CPS) and SonoVue. Eur Radiol 14 (Suppl 8):P53-P62

3. Schwartz LH, Gandras EJ, Colangelo SM, Ercolani MC, Panicek DM (1999) Prevalence and importance of small hepatic lesions found at $\mathrm{CT}$ in patients with cancer. Radiology 210(1):71-74

4. Khalil HI, Patterson SA, Panicek DM (2005) Hepatic lesions deemed too small to characterize at CT: prevalence and importance in women with breast cancer. Radiology 235(3):872-878

5. Gibbs JF, Litwin AM, Kahlenberg MS (2004) Contemporary management of benign liver tumors. Surg Clin North Am 84(2):463-480
6. Schneider G, Grazioli L, Saini S (2003) Imaging of benign focal liver lesions. In: Schneider G, Grazioli L, Saini S (eds) MRI of the liver, 1st edn. Springer-Verlag, Milan, Italy, pp 105-170

7. Vilgrain V, Boulos L, Vullierme MP, Denys A, Terris B, Menu Y (2000) Imaging of atypical hemangiomas of the liver with pathologic correlation. Radiographics 20(2):379-397

8. Anthony PP (1987) Tumors and tumor-like lesions of the liver and biliary tract, 2nd edn. In: MacSween RNM, Anthony PP, Scheuer PJ (eds) Pathology of the liver. Churchill Livingstone, New York

9. Mungovan JA, Cronan JJ, Vacarro J (1994) Hepatic cavernous hemangiomas: lack of enlargement over time. Radiology 191(1):111-113

10. Tung GA, Vaccaro JP, Cronan JJ, Rogg JM (1994) Cavernous hemangioma of the liver: pathologic correlation with high-field MR imaging. AJR Am J Roentgenol 162(2):1113-1117

11. Harvey CJ, Albrecht T (2001) Ultrasound of focal liver lesions. Eur Radiol 11(9):1578-1593
12. Jang H, Kim TK, Lim HK, Park SJ, Sim JS, Kim HY, Lee JH (2003) Hepatic hemangioma: atypical appearances on CT, MR imaging, and sonography. AJR Am J Roentgenol 180 (1):135-141

13. Kim S, Chung JJ, Kim MJ, Park SJ, Lee JT, Yoo HS (2000) Atypical insideout pattern of hepatic hemangiomas. AJR Am J Roentgenol 174(6):1571-1574

14. Bennett GL, Petersein A, Mayo-Smith WW, Hahn PF, Schima W, Saini S (2000) Addition of gadolinium chelates to heavily T2-weighted MR imaging: limited role in differentiating hepatic hemangiomas from metastases. AJR Am J Roentgenol 174(2):477-485

15. McFarland EG, Mayo-Smith WW, Saini S, Hahn PF, Goldberg MA, Lee MJ (1994) Hepatic hemangiomas and malignant tumors: improved differentiation with heavily T2-weighted conventional spin-echo MR imaging. Radiology 193(1):43-47 
16. Cieszanowski A, Szeszkowski W, Golebiowski M, Bielecki DK, Grodzicki M, Pruszynski B (2002) Discrimination of benign from malignant hepatic lesions based on their T2-relaxation times calculated from moderately T2-weighted turbo SE sequence. Eur Radiol 12(9):2273-2279

17. Chan YL, Lee SF, Yu SC, Lai P, Ching AS (2002) Hepatic malignant tumour versus cavernous haemangioma: differentiation on multiple breath-hold turbo spin-echo MRI sequences with different T2-weighting and T2-relaxation time measurements on a single slice multi-echo sequence. Clin Radiol 57(4):250-257

18. Fenlon HM, Tello R, deCarvalho VL, Yucel EK (2000) Signal characteristics of focal liver lesions on double echo T2-weighted conventional spin echo MRI: observer performance versus quantitative measurements of T2 relaxation times. J Comput Assist Tomogr 24(2):204-211

19. Ohkawa M, Katoh T, Nakano S, Fujiwara N, Mori Y, Hino I, Tanabe M (1997) Use of fluid-attenuated inversion recovery (FLAIR) pulse sequences for differential diagnosis of hepatic hemangiomas and hepatic cysts. Acta Med Okayama 51(5):275-278

20. Herborn CU, Vogt F, Lauenstein TC, Goyen M, Debatin JF, Ruehm SG (2003) MRI of the liver: can True FISP replace HASTE? J Magn Reson Imaging 17(2):190-196

21. Taouli B, Vilgrain V, Dumont E, Daire JL, Fan B, Menu Y (2003) Evaluation of liver diffusion isotropy and characterization of focal hepatic lesions with two single-shot echo-planar MR imaging sequences: prospective study in 66 patients. Radiology 226(1):71-78

22. Montet X, Lazeyras F, Howarth N, Mentha G, Rubbia-Brandt L, Becker CD, Vallee JP, Terrier F (2004) Specificity of SPIO particles for characterization of liver hemangiomas using MRI. Abdom Imaging 29(1):60-70

23. Zheng WW, Zhou KR, Chen ZW, Shen JZ, Chen CZ, Zhang SJ (2002) Characterization of focal hepatic lesions with SPIO-enhanced MRI. World J Gastroenterol 8(1):82-86
24. Bartolotta TV, Midiri M, Quaia E, Bertolotto M, Galia M, Cademartiri F, Lagalla R (2005) Liver haemangiomas undetermined at grey-scale ultrasound: contrast-enhancement patterns with SonoVue and pulse-inversion US. Eur Radiol 15(4):685-693

25. Lencioni R (2006) Impact of European Federation of Societies for Ultrasound in Medicine and Biology (EFSUMB) guidelines on the use of contrast agents in liver ultrasound. Eur Radiol 16 (7):1610-1613

26. Bartolotta TV, Midiri M, Quaia E Bertolotto M, Galia M, Cademartiri F, Lagalla R, Cardinale AE (2005) Benign focal liver lesions: spectrum of findings in SonoVue-enhanced pulse-inversion ultrasonography. Eur Radiol 15 (8):1643-1649

27. Colakoglu O, Taskiran B, Yazici N, Buyrac Z, Unsal B (2005) Safety of biopsy in liver hemangiomas. Turk $\mathrm{J}$ Gastroenterol 16(4):220-223

28. Yang DM, Kim HS, Cho SW, Kim HS (2002) Pictorial review: various causes of hepatic capsular retraction: CT and MR findings. Br J Radiol 75(900):994-1002

29. Lee SH, Park CM, Cheong IJ, Kwak MS, Cha SH, Choi SY, Kim CH (2001) Hepatic capsular retraction: unusual finding of cavernous hemangioma. $\mathrm{J}$ Comput Assist Tomogr 25(2):231-233

30. Bader TR, Braga L, Semelka RC (2001) Exophytic benign tumors of the liver: appearance on MRI. Magn Reson Imaging 19(5):623-628

31. Lapeyre M, Mathieu D, Tailboux L, Rahmouni A, Kobeiter H (2002) Dilatation of the intrahepatic bile ducts associated with benign liver lesions: an unusual finding. Eur Radiol 12(1):71-73

32. Ghai S, Dill-Macky M, Wilson S, Haider M (2005) Fluid-fluid levels in cavernous hemangiomas of the liver: baffled? AJR Am J Roentgenol 184(3 Suppl):S82-S85

33. Soyer P, Bluemke DA, Fishman EK, Rymer R (1998) Fluid-fluid levels within focal hepatic lesions: imaging appearance and etiology. Abdom Imaging 23(2):161-165

34. Obata S, Matsunaga N, Hayashi K, Ohtsubo M, Morikawa T, Takahara O (1998) Fluid-fluid levels in giant cavernous hemangioma of the liver: CT and MRI demonstration. Abdom Imaging 23(6):600-602
35. Kim KW, Kim TK, Han JK, Kim AY, Lee HJ, Choi BI (2001) Hepatic hemangiomas with arterioportal shunt: findings at two-phase CT. Radiology 219(3):707-711

36. Jeong MG, Yu JS, Kim KW (2000) Hepatic cavernous hemangioma: temporal peritumoral enhancement during multiphase dynamic MR imaging. Radiology 216(3):692-697

37. Yu JS, Kim KW, Park MS, Yoon SW (2002) Transient peritumoral enhancement during dynamic MRI of the liver: cavernous hemangioma versus hepatocellular carcinoma. J Comput Assist Tomogr 26(3):411-417

38. Giovagnoni A, Terilli F, Ercolani P, Paci E, Piga A (1994) MR imaging of hepatic masses: diagnostic significance of wedge-shaped areas of increased signal intensity surrounding the lesion. AJR Am J Roentgenol 163(5):1093-1097

39. Aibe H, Hondo H, Kuroiwa T, Yoshimitsu K, Irie H, Tajima T, Shinozaki K, Asayama Y, Taguchi K, Masuda K (2001) Sclerosed hemangioma of the liver. Abdom Imaging 26 (5):496-499

40. Brancatelli G, Federle MP, Blachar A, Grazioli L (2001) Hemangioma in the cirrhotic liver: diagnosis and natural history. Radiology 219(1):69-74

41. Dodd GD 3rd, Baron RL, Oliver JH 3rd, Federle MP (1999) Spectrum of imaging findings of the liver in endstage cirrhosis. II. Focal abnormalities. AJR Am J Roentgenol

173(5):1185-1192

42. Mastropasqua M, Kanematsu M, Leonardou P, Braga L, Woosley JT, Semelka RC (2004) Cavernous hemangiomas in patients with chronic liver disease: MR imaging findings. Magn Reson Imaging 22(1):15-18

43. Mathieu D, Zafrani ES, Anglade MC, Dhumeaux D (1989) Association of focal nodular hyperplasia and hepatic hemangioma. Gastroenterology 97 (1):154-157

44. Vilgrain V, Uzan F, Brancatelli G, Federle MP, Zappa M, Menu Y (2003) Prevalence of hepatic hemangioma in patients with focal nodular hyperplasia: MR imaging analysis. Radiology 229 (1):75-79 